\title{
Spatially Resolved Characterization of the Coherence Area in the Incoherent Emission Regime of a Broad-Area Vertical-Cavity Surface-Emitting Laser
}

\author{
Guy Verschaffelt, Gordon Craggs, Michael L. F. Peeters, Shyam K. Mandre, Hugo Thienpont, Member, IEEE, \\ and Ingo Fischer
}

\begin{abstract}
We present direct measurements of the spatial coherence area of a pulsed broad-area vertical-cavity surface-emitting laser using a reversing wavefront interferometer. With this technique, we can assess the size and uniformity of the coherence area across the laser aperture, being of importance for projection applications. We show that the output beam can be considered quasi-homogeneous and that the measured coherence area corresponds well with the coherence area deduced from the far-field emission profile. We demonstrate that the coherence area is limited in size by the radial temperature gradient in the device and discuss the origin of coherence variations.
\end{abstract}

Index Terms-Semiconductor laser, spatial coherence, verticalcavity surface-emitting laser (VCSEL).

\section{INTRODUCTION}

$\mathbf{V}$ ERTICAL-CAVITY surface-emitting lasers (VCSELs) find their use nowadays in a number of applications such as optical data communication and sensing. If required, these lasers can provide a single-mode circular beam at low cost. To obtain such a high-quality beam, it is necessary to restrict the transverse size of the cavity to a few micrometers. The small transverse size will limit the available output power to a few milliwatts typically [1]. This low output power level makes small-aperture VCSELs unsuitable for a number of applications such as illumination, projection, and printing. Then, the output power can easily be increased by raising the transverse size of the cavity [2]. This comes, however, at the expense of having a much reduced beam-quality: because of the large Fresnel

Manuscript received May 14, 2008; revised September 01, 2008. Current version published March 11, 2009. This work was supported in part by the IAP P6/10 network "Photonics@be" and the VUB-IOF project "Micro-photonics".

G. Verschaffelt, G. Craggs, and H. Thienpont are with the Department of Applied Physics and Photonics, Vrije Universiteit Brussel, 1050 Brussels, Belgium (e-mail: guy.verschaffelt@vub.ac.be; gordon.craggs@vub.ac.be; hthienpo@vub.ac.be).

M. L. F. Peeters was with the Department of Applied Physics and Photonics, Vrije Universiteit Brussel, 1050 Brussels, Belgium. He is now with AlcatelLucent, 2018 Antwerpen, Belgium (e-mail: m.l.f.peeters@gmail.com).

S. K. Mandre was with the Institute of Applied Physics, Darmstadt University of Technology, D-64289 Darmstadt, Germany. He is now with Braun GmbH, 61476 Kronberg, Germany (e-mail: shyammandre@google-mail.com).

I. Fischer was with the Department of Applied Physics and Photonics, Vrije Universiteit Brussel, 1050 Brussels, Belgium. He is now with the Joint Research Institute of Integrated Systems and the School of Engineering and Physical Sciences, Heriot-Watt University, Edinburgh EH14 4AS, U.K. (e-mail: laserdynamics@gmail.com).

Digital Object Identifier 10.1109/JQE.2009.2013085 number of such broad-area VCSELs (BA-VCSEL), multiple competing transverse modes will be emitted [3], [4].

Besides emission in multiple transverse modes, we have shown [5] that one can obtain a drastically different emission regime with BA-VCSELs by driving these devices with $\mu$ s-pulses. Using the proper pulse amplitude, the BA-VCSEL will no longer emit globally defined transverse modes but instead it will emit a nonmodal, spatially incoherent beam. This incoherent emission can be understood as emission in multiple, independent, spatially separated coherence islands. In [6], we have shown that there is a dynamic transition during the first few hundreds of nanoseconds of the pulse from modal to incoherent emission. This transition takes place on the thermal time-scale of the VCSEL. During this transition, the far-field intensity profile evolves from a modal, structured pattern into a Gaussian shaped profile. At the same time, modal structures disappear in the optical spectrum. The observed loss of spatial coherence is not accompanied by any noticable reduction of the output power.

This high-power, spatially incoherent emission regime is quite uncommon for semiconductor lasers but can be useful in, e.g., illumination and projection applications as the low degree of spatial coherence may help to reduce speckle [7]. Therefore, this emission regime deserves further study. In this paper, we present measurements of the spatial coherence area for different positions in the VCSEL aperture using a reversing wavefront interferometer. We show that the coherence area is much smaller than the VCSEL aperture and that the intensity is uniform over the scale of the coherence area. This further substantiates the conjecture put forward in [5] that a BA-VCSEL can be operated as a quasi-homogeneous source. We also show that the spatially and intensity weighted average of the measured coherence radius corresponds well with the coherence radius deduced from the far-field divergence angle. Finally, we will discuss in how far the measured coherence radius is limited by the quasi-mode size of a planar cavity.

\section{Device Characteristics}

In our measurements, we use an oxide confined BA-VCSEL with an aperture radius $R$ of $25 \mu \mathrm{m}$, similar to the device used in [5]. The BA-VCSEL emits at a wavelength of $\sim 840 \mathrm{~nm}$, with a maximum $\mathrm{CW}$ output power of $\sim 70 \mathrm{~mW}$ at an injected current of $80 \mathrm{~mA}$. The VCSEL's threshold current is approximately $14 \mathrm{~mA}$. In our experiments, we drive the VCSEL with a pulsed current of $240-\mathrm{mA}$ amplitude, $1-\mu$ s pulse length, and a duty 


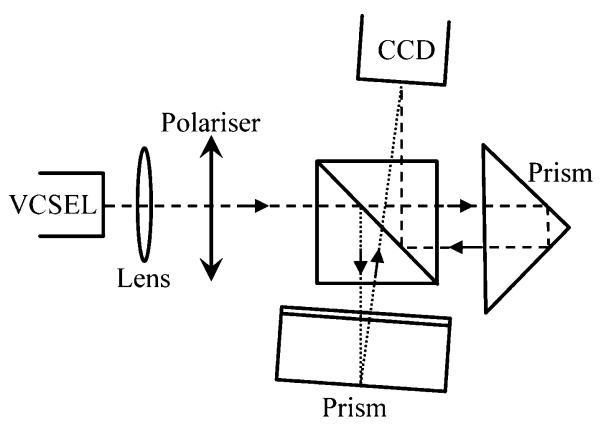

Fig. 1. Schematic drawing of the reversing wavefront Michelson interferometer.

cycle of $0.5 \%$. These pulse conditions induce the nonmodal, incoherent emission regime [5].

\section{SETUP}

We use a wavefront reversing Michelson interferometer [8], [9] to measure the spatial coherence properties of the VCSEL. The scheme of this interferometer is shown in Fig. 1. The VCSEL's near-field (NF) is imaged onto the CCD-camera with a magnification of 120 . A linear polarizer is inserted into the beam path in order to select one polarization direction. The beam traveling towards the CCD camera is split into two parts with a 50/50 nonpolarizing beam splitter. In each arm of the interferometer, a right-angle prism is used to reflect the beam. In one arm, the beam is flipped horizontally while being reflected by the prism, whereas in the other arm the beam is flipped vertically. Therefore, the two beams interfering at the CCD are effectively spatially rotated $180^{\circ}$ with respect to each other. In our setup, we thus measure the correlation between radially symmetric points. To avoid image distortion that occurs when the beam hits the apex of the right-angle prisms, the beams are directed off axis with respect to the prism-apex. As a consequence, the two prisms have to be slightly tilted in order to overlap the beams at the CCD-camera. This tilt is also used to control and vary a relative vertical offset between the two interfering beams. This way, we choose the center of the spatial interferogram. In order to obtain an equal path length in both arms, we laterally move the prism in one of the arms until we attain a maximum fringe visibility at the correlation measurement's center. One of the prisms is mounted on a piezoelectric-actuator through which the path-length difference between the two arms can be changed with subwavelength resolution.

Employing this setup, the complex degree of coherence $\mu$ can be deduced by recording four images. First, we record the interference pattern $I_{t}^{0}$ when both arms of the interferometer have equal lengths. Next, we measure the interference pattern $I_{t}^{\lambda / 4}$ when one of the arms is shifted by a quarter wavelength. We subsequently record the intensity distribution $I_{0}^{H}$ when one arm is blocked and $I_{0}^{V}$ when the other arm is blocked. Finally, the
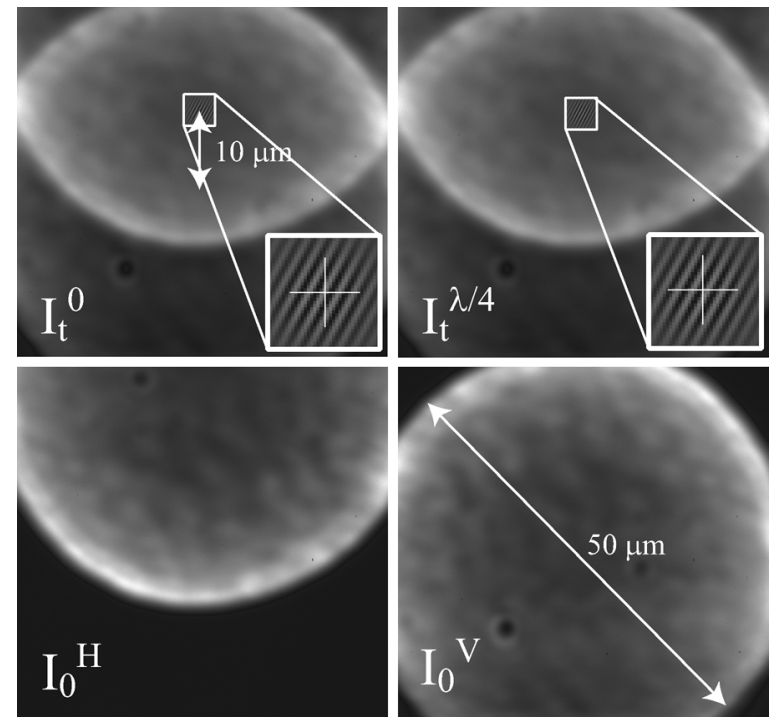

Fig. 2. Images $I_{t}^{0}, I_{t}^{\lambda / 4}, I_{0}^{H}$, and $I_{0}^{V}$ for a shift of $10 \mu \mathrm{m}$ between the VCSEL's center and the correlation measurement's center. The insets of the two images at the top show a $3 \mu \mathrm{m} \times 3 \mu \mathrm{m}$ region of $I_{t}^{0}$ and $I_{t}^{\lambda / 4}$ around the correlation measurement's center (indicated by the white crosshairs).

magnitude of the complex degree of coherence can be calculated via [9]

$$
|\mu|=\frac{\left\{\left[I_{t}^{0}-I_{0}^{H}-I_{0}^{V}\right]^{2}+\left[I_{t}^{\lambda / 4}-I_{0}^{H}-I_{0}^{V}\right]^{2}\right\}^{1 / 2}}{2 \sqrt{I_{0}^{H} I_{0}^{V}}} .
$$

An example of the four required images $I_{t}^{0}, I_{t}^{\lambda / 4}, I_{0}^{H}$, and $I_{0}^{V}$ is presented in Fig. 2 for a 2.4-mm vertical shift between the two beams. This corresponds to a shift of $20 \mu \mathrm{m}$ expressed in the VCSEL's NF coordinates. The central point of the correlation measurements (i.e., the center of the spatial interferogram) is therefore not the middle of the VCSEL aperture, but it is shifted $10 \mu \mathrm{m}$ upward from the center of the VCSEL. This shift can be used to probe the complex degree of coherence at various points within the VCSEL aperture. In the inset of Fig. 2 for $I_{t}^{0}$, one can see that the correlation measurement's center (indicated by the white crosshair) coincides with a maximum of the interference fringes. For $I_{t}^{\lambda / 4}$, the intensity at the correlation measurement's center is halfway between a minimum and maximum of the interference fringes, indicating that the shift between the measured images for $I_{t}^{0}$ and $I_{t}^{\lambda / 4}$ indeed corresponds to a quarter wavelength.

The $I_{0}^{H}$ and $I_{0}^{V}$ images clearly show that the intensity is not uniform across the VCSEL aperture. The intensity is higher at the edge of the VCSEL aperture due to current crowding. Interference fringes are only visible in the $I_{t}^{0}$ image of Fig. 2 close to the central point. This means the correlations have a rather limited spatial extent. This limited spatial coherence length becomes more readily visible if we use (1) to calculate the magnitude of the complex degree of coherence. We perform this detailed analysis in Section IV. 


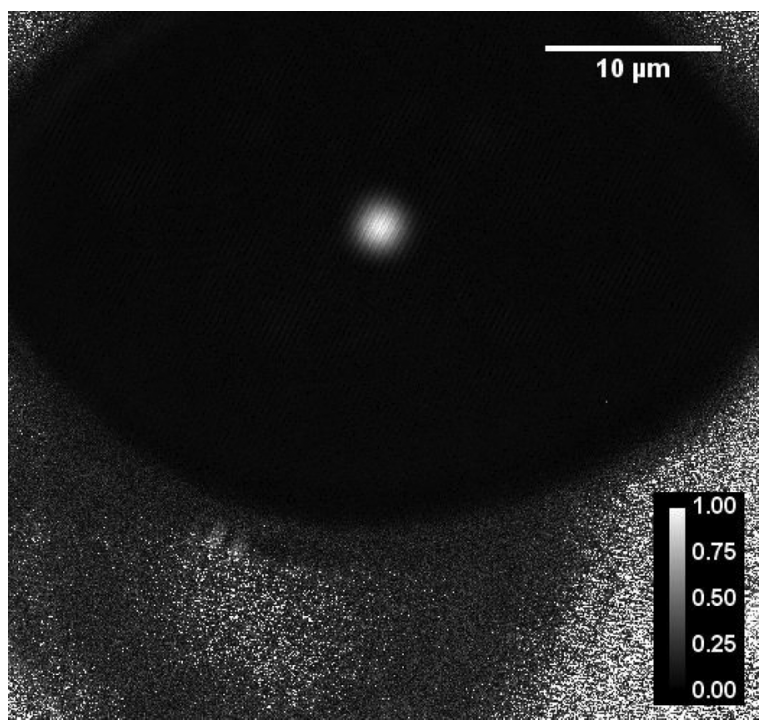

Fig. 3. Magnitude of the complex degree of coherence $|\mu|$ in pulsed operation (nonmodal emission) for a shift of $10 \mu \mathrm{m}$ between the VCSEL's center and the correlation measurement's center.

\section{DEGREe OF COHERENCE}

In Fig. 3, we plot an intensity map of $|\mu|$ obtained by applying (1) on the images shown in Fig. 2. Equation (1) is only valid for those positions in the image plane where the beams originating from both interferometer arms overlap. Therefore, the values at the outer edges of Fig. 3 are determined by noise and can be disregarded. Where the two beams do overlap in Fig. 2, we see in Fig. 3 that $|\mu|$ is only large close to the correlation measurement's center. This is in stark contrast to what we obtain in case of modal emission: each of the transverse modes is fully coherent over itself, which results in interference fringes and a non-zero value for $|\mu|$ all over the VCSEL aperture. This is illustrated in Fig. 4, where we plot the magnitude of the complex degree of coherence when the VCSEL is operated at a CW current of $40 \mathrm{~mA}$. At this $\mathrm{CW}$ current, the VCSEL emits a multitude of transverse modes which partly overlap with each other. As a result, we see in Fig. 4 that $|\mu|$ is nonzero at most positions across the area of the VCSEL's emission, exhibiting a complex pattern of mutually coherent spots.

Fig. 3 also clearly illustrates that $|\mu|$ is circularly symmetric. We characterize the extent of $|\mu|$ using the coherence radius $\xi$ at which $|\mu|$ has decreased by $1 / e^{2}$ compared to its value at the correlation measurement's center. To estimate the value of $\xi$ and at the same time investigate the shape of $|\mu|$, we plot in Fig. 5 a transverse cut of Fig. 3 through the correlation measurement's center. In principle, $|\mu|$ should be 1 at the center (i.e., at position $=0 \mu \mathrm{m}$ in Fig. 5). The fact that we obtain a maximum value of 0.9 for $|\mu|$ is due to experimental restrictions, most notably the finite resolution of the imaging system and the mechanical stability of the setup. In Fig. 5, we also compare the measured complex degree of coherence with a fit to a Gaussian function, which allows us to determine the coherence radius $\xi$, yielding a value of $1.91 \mu \mathrm{m}$. The coherence radius is thus clearly much smaller than the VCSEL's aperture radius of $25 \mu \mathrm{m}$.

We repeated the above described measurement of $|\mu|$ for different positions of the correlation measurement's center

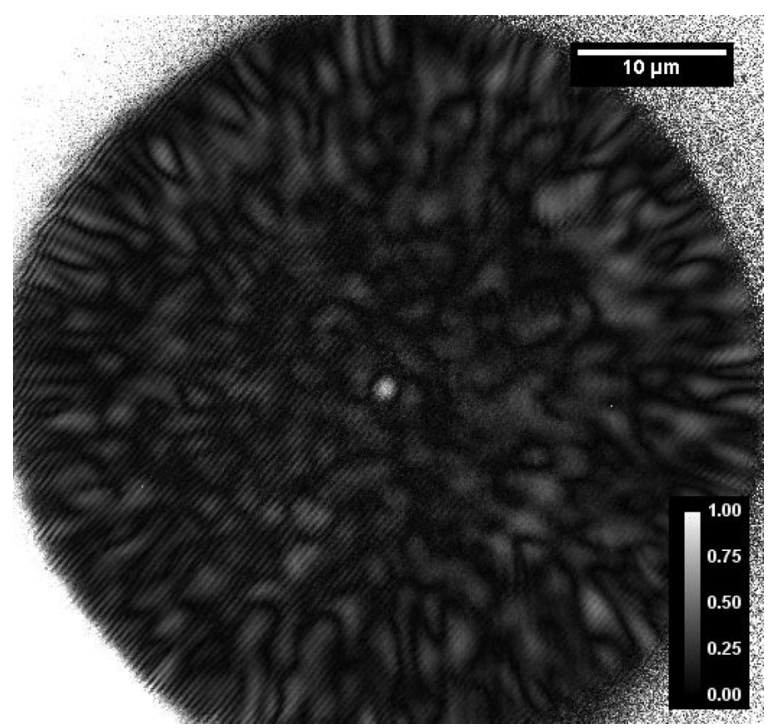

Fig. 4. Magnitude of the complex degree of coherence $|\mu|$ in $\mathrm{CW}$ operation for an injected current of $40 \mathrm{~mA}$ (modal emission) and no shift between the VCSEL's center and the correlation measurement's center.

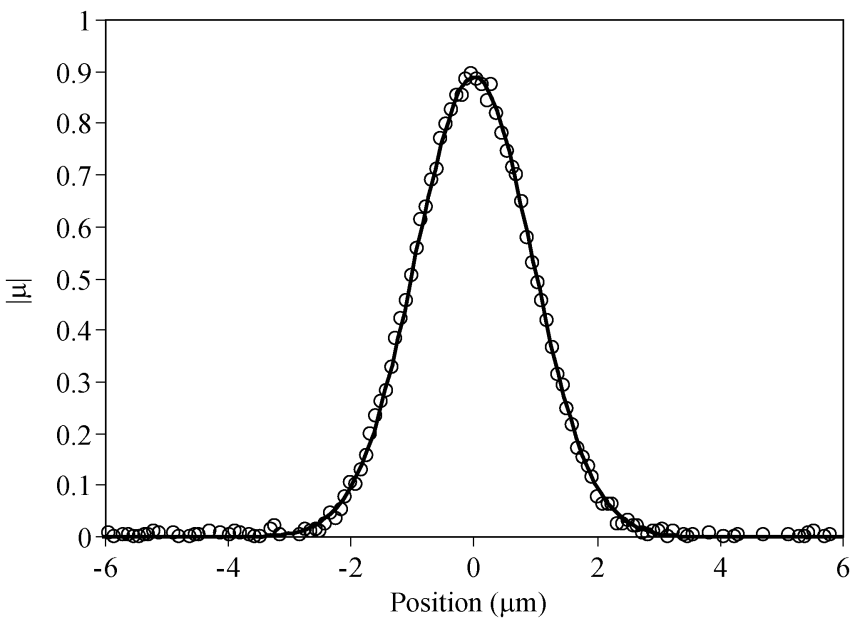

Fig. 5. Transverse cut of the magnitude of the complex degree of coherence $|\mu|$ shown in Fig. 3 (open circles) and fit to a Gaussian function (full line).

within the VCSEL aperture. The values of the coherence radius $\xi$ that we extracted from these measurements are plotted in Fig. 6 versus the radial position within the VCSEL aperture. This figure shows that the coherence radius $\xi$ is not constant across the entire VCSEL aperture, but that it decreases towards the rim of the device. The intensity and area weighted average of the coherence area is given by

$$
\xi_{\text {average }}=\frac{\int_{0}^{R} \xi(r) P(r) r d r}{\int_{0}^{R} P(r) r d r}
$$

where $r$ is the radial coordinate in the VCSEL aperture and $P(r)$ is the radial intensity distribution. The measurements shown in Fig. 6 yield an average coherence radius $\xi_{\text {average }}$ of $1.4 \mu \mathrm{m}$. The coherence radius remains in between 1 and $2 \mu \mathrm{m}$ for all positions. If we compare this to the NF intensity distribution (see, e.g., $I_{0}^{V}$ in Fig. 2), we can see that the intensity does not 


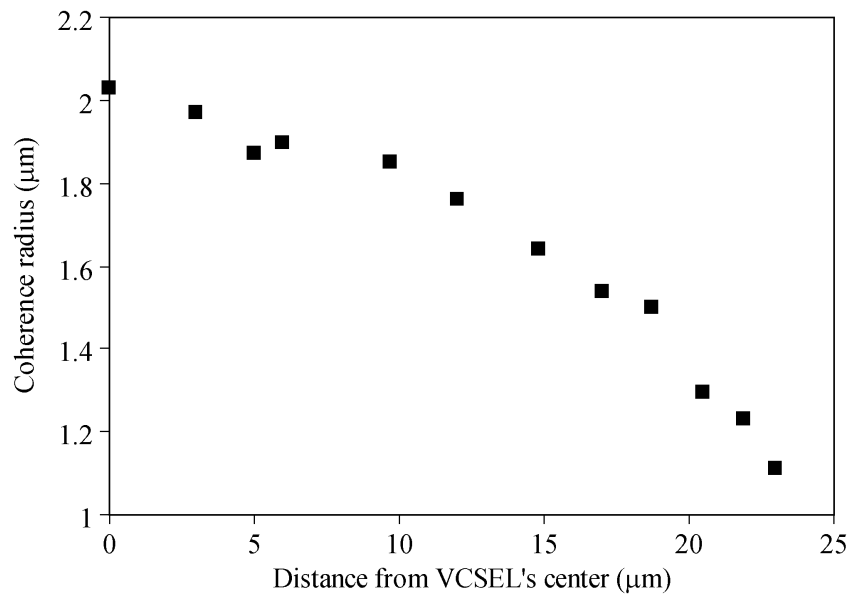

Fig. 6. Coherence radius $\xi$ as a function of the position within the VCSEL aperture.

change drastically over distances of the dimension of the coherence radius. Therefore, we can indeed consider the source to be quasi-homogeneous [10] with a Gaussian-shaped coherence function, i.e.,

$$
\mu(\rho)=\exp \left(-2 \rho^{2} / \xi^{2}\right)
$$

where $\rho$ is the NF radial coordinate.

With this knowledge, we can now make the link between the complex degree of coherence discussed above and the measurements of the far-field profile discussed in [5]. For a similar device and driving conditions as described in this paper, we reported in [5] that the BA-VCSEL no longer shows a structured modal far-field. Instead a Gaussian-shaped far-field intensity distribution is observed with a half opening angle (at $1 / \mathrm{e}^{2}$ ) of $11^{\circ}$. For a light source with arbitrary spatial coherence, the intensity distribution $J(\vec{s})$ in the far-field direction $\vec{s}$ can be determined from the source's complex degree of coherence $\mu(\vec{\rho})$ via [10]-[12]

$$
J(\vec{s}) \propto \cos ^{2} \theta \int_{A} \mu(\vec{\rho}) C(\vec{\rho}) e^{i \vec{f} \cdot \vec{\rho}} d A
$$

where the integration is performed over the near-field coordinate $\vec{\rho}$ and over the aperture area $A$. $C(\vec{\rho})$ denotes the autocovariance of the source's aperture amplitude function. The spatial frequency $\vec{f}$ is related to the projection $\vec{s}_{\perp}$ of the direction $\vec{s}$ on the observation plane, where $\vec{f}=(2 \pi \lambda) / \vec{s}_{\perp}$. The angle of $\vec{s}$ with respect to the beam center is given by $\theta$.

For a quasi-homogeneous source with a Gaussian coherence function - which is a good approximation for our device- the far-field intensity distribution resulting from (4) is given by [10]

$$
\begin{aligned}
J(\theta) & =J(0) \cos ^{2}(\theta) \exp \left[2 \frac{\pi^{2} \xi^{2}}{\lambda^{2}} \sin ^{2}(\theta)\right] \\
& \approx J(0) \exp \left(-2 \theta^{2} / \theta_{1 / e^{2}}^{2}\right)
\end{aligned}
$$

where the approximate form is valid for paraxial angles. The far-field divergence angle $\theta_{1 / e^{2}}$ is thus related to the coherence radius $\xi$ via

$$
\theta_{1 / e^{2}}=\frac{\lambda}{\pi \xi}
$$

If we use (6) together with the measured half-width divergence angle (at $1 / \mathrm{e}^{2}$ ) of $11^{\circ}$, we find a coherence radius of $1.4 \mu \mathrm{m}$. This is in excellent agreement with the average coherence radius $\xi_{\text {average }}$ of $1.4 \mu \mathrm{m}$ that we obtained from the reversing wavefront experiments.

\section{QuASI-Mode SIZE}

In the previous sections, we have shown that our pulsed BA-VCSEL is indeed a partially coherent quasi-homogeneous source with a Gaussian-shaped coherence function. After investigating the dynamic transition in the near- and far-field, we were able to conclude in [6] that the loss of modal emission is due to the fast thermal chirp of the VCSEL's cavity during the pulse, which-in combination with a spatially distributed thermal lens - prevents the build-up of cavity modes. However, this does not explain why we obtain a coherence radius of approximately $1.4 \mu \mathrm{m}$ or, equivalently, a far-field divergence angle of $11^{\circ}$.

To understand by which mechanisms the coherence area is being limited, let us consider the approach taken by [13]-[15]. This approach defines the mode size in a planar cavity, taking into account diffraction in the cold cavity. Starting from a planar cavity implies neglecting the boundary conditions imposed by the oxide aperture at the outer rim of the device. This seems to be a good approximation since the coherence radius $\xi$ is much smaller than the device's aperture radius $R$. The assumption of a cold cavity neglects the influence of spatial carrier distribution. In a further stage, we will only consider the presence of a spatially distributed thermal lens caused by the inhomogeneous current injection and heating of the VCSEL cavity. In addition, dynamical effects are neglected, as a full dynamical study requires microscopic modeling of the semiconductor medium and a spatially resolved treatment of the cavity [16], [17]. This is beyond the scope of this manuscript. Nevertheless, the consideration presented here provides an upper limit for the expected coherence radii and allows to evaluate the importance of the considered mechanism.

The mode size $a_{p}$ in a planar cavity is given by the lateral speed of the photons in the cavity multiplied by the photon lifetime [15], resulting in

$$
a_{p} \approx \sqrt{\frac{\lambda L}{\pi n_{\mathrm{cav}}\left(1-\sqrt{R_{1} R_{2}}\right)}}
$$

where $\lambda$ is the vacuum wavelength, $L$ is the effective cavity length, $n_{\text {cav }}$ is the refractive index of the cavity, and $R_{1,2}$ is the reflectivity of the top and bottom DBR of the VCSEL. This mode has a linewidth $\Delta \lambda$ of [13]

$$
\Delta \lambda \approx \frac{\lambda^{2}\left(1-\sqrt{R_{1} R_{2}}\right)}{2 \pi L n_{\text {cav }}} .
$$




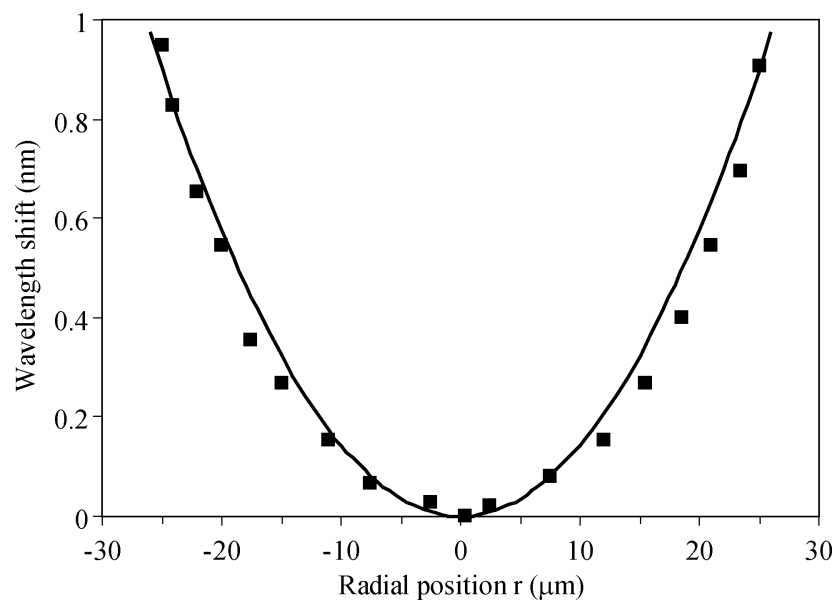

Fig. 7. Radial wavelength shift relative to the wavelength in the center of the BA-VCSEL for $1-\mu$ s pulses with an amplitude of $160 \mathrm{~mA}$ (squares) and comparison to a parabolic fit (full line).

From (7) and (8), it can be deduced that the mode area is inversely proportional to the linewidth of that mode

$$
a_{p}^{2} \approx \frac{\lambda^{3}}{2 \pi^{2} n_{\text {cav }}^{2}} \frac{1}{\Delta \lambda}
$$

Equation (7) has been derived for a planar cavity. For our experimental driving conditions, the VCSEL exhibits a considerable gradient in refractive index because of inhomogeneous current injection and Joule heating [18]. We have been able to determine the spatially resolved thermal gradient of the VCSEL by measuring the spectrally dispersed NF profile [18]: when the VCSEL is driven in the regime of spatially incoherent emission, the local wavelength shift can be attributed to local temperature changes. In Fig. 7, we show the measured spatially resolved wavelength shift (relative to the wavelength in the center of the device) for a pulse amplitude of $160 \mathrm{~mA}$ and length of $1 \mu \mathrm{s}$. The thermal wavelength shift $\Delta \lambda_{\text {thermal }}$ within the cavity can be described by a parabola, i.e., $\Delta \lambda_{\text {thermal }}=B r^{2}$ with $B$ being $1.4 \times 10^{-6} \mu \mathrm{m}^{-1}$ for a pulse amplitude of $160 \mathrm{~mA}$. In [18], we have shown that the radial wavelength shift is proportional to the power dissipated in the VCSEL. Based on the VCSEL's steady-state characteristics, we estimate the power dissipation to be $390 \mathrm{~mW}$ at an injected current of $160 \mathrm{~mA}$ and $780 \mathrm{~mW}$ at an injected current of $240 \mathrm{~mA}$. We thus estimate the radial wavelength shift for the pulsed driving conditions discussed in this paper to correspond to a value for $B$ of $2.7 \times 10^{-6} \mu \mathrm{m}^{-1}$.

We have to take into account the effect of the thermal wavelength shift as soon as it becomes comparable to the linewidth of the modes as described by (9). In particular, if the mode size $a_{p}$ is large, the thermal wavelength shift over the mode will be nonnegligible compared with the linewidth $\Delta \lambda$ as expressed by (8). This further reduces the spatial coherence radius or mode size. Therefore, we redefine the mode size as being the spatial extent across which the thermal wavelength shift $\Delta \lambda_{\text {thermal }}$ is small compared with the linewidth $\Delta \lambda$ :

$$
\Delta \lambda_{\text {thermal }} \leq \gamma \Delta \lambda .
$$

The parameter $\gamma$ describes the wavelength shift at which the visibility of an interferogram (that originates from two Lorentzian

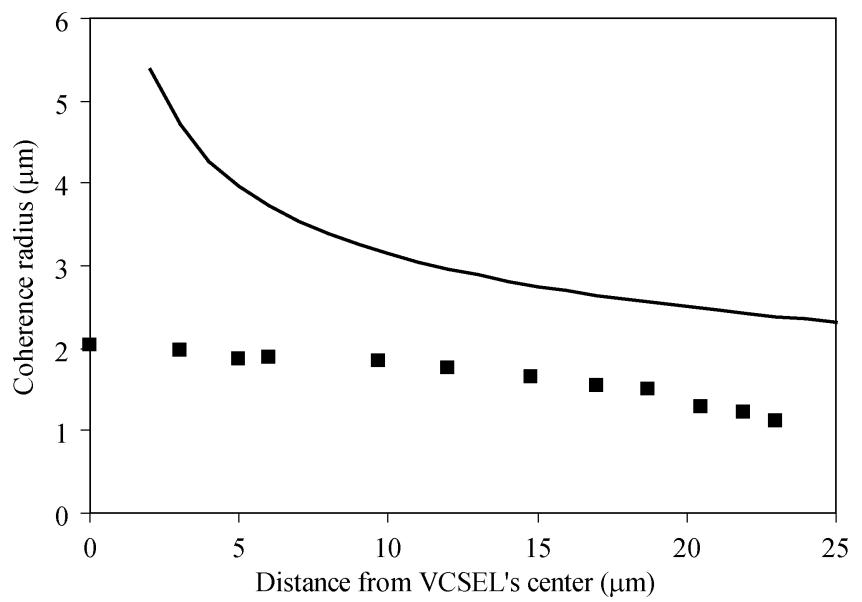

Fig. 8. Coherence radius $\xi$ as a function of the position within the VCSEL aperture (squares) and comparison with the quasi-mode size from (11) (full line).

line shapes of the same linewidth) has dropped to $1 / e^{2}$. From this, we obtain a value of $\gamma=\sqrt{e-1} \cdot \Delta \lambda=1.3 \cdot \Delta \lambda$. If we use (9) to further elaborate this condition, we finally obtain an upper estimate for the mode size $a_{p}$

$$
a_{p} \approx \frac{\lambda}{2} \sqrt[3]{\frac{\gamma}{\pi^{2} n_{\mathrm{cav}}^{2} B r}}
$$

This mode size is plotted in Fig. 8, together with the measured coherence radius for different radial positions. We obtain an upper limit for the coherence radii of the order of $4 \mu \mathrm{m}$. This is already one order of magnitude smaller than the laser aperture, indicating that the spatial coherence properties have significantly changed compared to modal emission. We note that the measured coherence radii are still about a factor of two smaller than this upper limit. This indicates that additional mechanisms, including the aforementioned spatial carrier distribution and dynamical effects, further contribute to the decrease of the mode size. Nevertheless, our simplified considerations illustrate the limits of the mode-size imposed by diffraction in a cavity with a strong refractive index profile. According to our estimates the coherence radius increases strongly towards the center of the VCSEL's aperture. This is in contrast to our experimental findings where the coherence radius is homogeneous across the device aperture, with only a slight decay towards the rim. The origin of this is still unclear, however, we emphasize that the behavior of the VCSEL's central part has a small effect on the overall characteristics of the device: it will only contribute to the total emission for a small amount as both the emitted power density and the emission area are smallest in this region.

The mode size resulting from (11) is only weakly dependent on the thermal gradient $B$. This gradient depends on the VCSEL's structure and the pulse parameters. Therefore, we expect the mode size to be only weakly dependent on the epitaxial structure, the pulse amplitude, and the pulse duration. To check the dependence of the mode size on the pulse parameters, we measured the far-field divergence angle in the spatially incoherent emission regime for different pulse durations (from 1 to $4 \mu \mathrm{s}$ ) and pulse amplitudes (from 140 to $300 \mathrm{~mA}$ ). In all cases, we obtained a half width divergence angle between $10^{\circ}$ 
and $13^{\circ}$. Using (6), this range corresponds to an average coherence radius between 1.2 and $1.5 \mu \mathrm{m}$. The coherence radius is thus not strongly dependent on the pulse parameters. Furthermore, the mode size $a_{p}$ is independent of the VCSEL's aperture diameter. Again, we checked this by measuring the far-field divergence angle in the spatially incoherent emission regime for devices with the same structure but different aperture diameters (ranging from 20 to $50 \mu \mathrm{m}$ ). The extracted coherence radius is in the range between 1.2 and $1.5 \mu \mathrm{m}$, demonstrating the independence of $a_{p}$ on the VCSEL's aperture diameter. The detailed measurement of the complex degree of coherence for different driving conditions and different device structures is a lengthy process and is beyond the scope of this paper.

\section{CONCLUSION}

We have used a $180^{\circ}$ reversing wavefront Michelson interferometer to measure the complex degree of coherence of a BA-VCSEL with an aperture diameter of $50 \mu \mathrm{m}$. The BA-VCSEL was driven into a spatially incoherent emission regime that can be obtained by strongly pulsing these devices. Our measurements show that in this regime the VCSEL can be considered a quasi-homogeneous source with a Gaussian shaped coherence function. The extracted coherence radius varies slightly within the VCSEL's aperture, but always remains small compared to the aperture size. The Gaussian shape of the coherence function manifests itself in a Gaussian intensity distribution in the far-field. The average coherence area of 1.4 $\mu \mathrm{m}$ corresponds well with the measured far-field divergence angle of 11 degrees. Our approach can also be used to measure the spatial coherence properties of other sources and might, e.g., be useful to measure the build-up of spatial coherence in coherent VCSEL arrays [19].

We have discussed the limitations imposed on the mode size because of the thermal gradient within the VCSEL aperture. The thermal gradient results in a radial wavelength shift that prevents the modes to grow beyond a few microns. The derived mode size is only weakly dependent on the pulse parameters and the VCSEL's structure. The proposed model can not fully explain the particular size of the measured coherence area, but rather it provides an upper limit for the coherence area. In practice, the measured coherence area is even smaller and much more uniform across the VCSEL aperture than expected from the model, which is promising for applications utilising the VCSEL's quasi-homogeneous properties.

These results are important to better understand the behavior of BA-VCSELs as partially coherent light sources. Such highpower primary sources of spatially incoherent radiation can be useful as the low degree of spatial coherence may help to reduce speckle. The small size of the coherence radius is beneficial for speckle reduction, as each spatially uncorrelated region can in principle lead to the formation of an independent speckle pattern. The optimum speckle reduction factor is therefore inversely proportional to the coherence radius. Our results also indicate that this kind of emission regime should be possible in other types of laser structures: the necessary ingredients are a strong transversal gradient in the cavity length or refractive index together with a fast chirp to keep the laser in a transient state. In order to further validate this argument, future studies will be aimed at estimating the modal build-up time and calculating how it is linked to a transverse gradient of the refractive index.

\section{ACKNOWLEDGMENT}

The authors would like to thank M. Grabherr of U-L-M Photonics $\mathrm{GmbH}$ for providing excellent BA-VCSELs.

\section{REFERENCES}

[1] A. Haglund, J. Gustavsson, J. Vukusic, P. Modh, and A. Larsson, "Single fundamental-mode output power exceeding $6 \mathrm{mw}$ from VCSELs with a shallow surface relief," IEEE Photon. Technol. Lett., vol. 16, no. 2, pp. 368-370, Feb. 2004

[2] M. Miller, M. Grabherr, R. King, R. Jäger, R. Michalzik, and K. J. Ebeling, "Improved output performance of high-power VCSELs," IEEE J. Sel. Top. Quantum Electron., vol. 7, no. 2, pp. 210-216, Mar.-Apr. 2001.

[3] C. J. Chang-Hasnain, M. Orenstein, A. Von Lehmen, L. T. Florez, J. P. Harbison, and N. G. Stoffel, "Transverse mode characteristics of vertical cavity surface-emitting lasers," Appl. Phys. Lett., vol. 57, pp. 218-220, 1990.

[4] C. Degen, I. Fischer, and W. Elsäßer, "Transverse modes in oxide confined VCSELs: Influence of pump profile, spatial hole burning, and thermal effects," Opt. Exp., vol. 5, pp. 38-47, 1999.

[5] M. Peeters, G. Verschaffelt, H. Thienpont, S. K. Mandre, I. Fischer, and M. Grabherr, "Spatial decoherence of pulsed broad-area vertical-cavity surface-emitting lasers," Opt. Exp., vol. 13, pp. 9337-9345, 2005.

[6] S. K. Mandre, W. Elsäßer, I. Fischer, M. Peeters, and G. Verschaffelt, "Evolution from modal to spatially incoherent emission of a broad-area VCSEL," Opt. Exp., vol. 16, pp. 4452-4464, 2008.

[7] J. Goodman, Speckle Phenomena in Optics: Theory and Applications. Greenwood Village, CO: Roberts \& Co., 2006.

[8] M. Santarsiero and R. Borghi, "Measuring spatial coherence by using a reversed-wavefront young interferometer," Opt. Lett., vol. 31, pp. 861-863, 2006.

[9] M. Imai, Y. Ohtsuka, and S. Satoh, "Spatial coherence analysis of light propagation in optical fibers by interferometric methods," J. Opt. Soc. Amer. A, vol. 3, pp. 1059-1064, 1986.

[10] L. Mandel and E. Wolf, Optical Coherence and Quantum Optics. Cambridge, U.K.: Cambridge Univ., 1995.

[11] A. C. Schell, "A technique for the determination of the radiation pattern of a partially coherent aperture," IEEE Trans. Antennas Propag., vol. AP-15, no. 1, pp. 187-188, Jan. 1967.

[12] E. Collett and E. Wolf, "Is complete spatial coherence necessary for the generation of highly directional light beams," Opt. Lett., vol. 2, pp. 27-29, 1978.

[13] G. Björk, H. Heitmann, and Y. Yamamoto, "Spontaneous-emission coupling factor and mode characteristics of planar dielectric microcavity lasers," Phys. Rev. A, vol. 47, pp. 4451-4463, 1993.

[14] F. De Martini, M. Marrocco, and D. Murra, "Transverse quantum correlations in the active microscopic cavity," Phys. Rev. Lett., vol. 65, pp. 1853-1856, 1990.

[15] H. Deng, Q. Deng, D. Deppe, D. Huffaker, and J. Shin, "Transverse and temporal mode dependence on mirror contrast in microcavity lasers," IEEE J. Quantum Electron., vol. 31, no. 12, pp. 2026-2036, Dec. 1995.

[16] E. Gehrig, O. Hess, and R. Wallenstein, "Modeling of the performance of high-power diode amplifier systems with an optothermal microscopic spatio-temporal theory," IEEE J. Quantum Electron., vol. 35, no. 3, pp. 320-331, Mar. 1999.

[17] M. Kolesik and J. Moloney, "A spatial digital filter method for broadband simulation of semiconductor lasers," IEEE J. Quantum Electron., vol. 37, no. 7, pp. 936-944, Jul. 2001.

[18] S. K. Mandre, W. Elsäßer, I. Fischer, M. Peeters, and G. Verschaffelt, "Determining the temporally and radially resolved temperature distribution inside a pulsed broad-area vertical-cavity surface-emitting laser cavity," Appl. Phys. Lett., vol. 89, p. 151 106, 2006.

[19] L. Lundeberg, G. Lousberg, D. Boiko, and E. Kapon, "Spatial coherence measurements in arrays of coupled vertical cavity surface emitting lasers," Appl. Phys. Lett., vol. 90, p. 021103, 2007. 


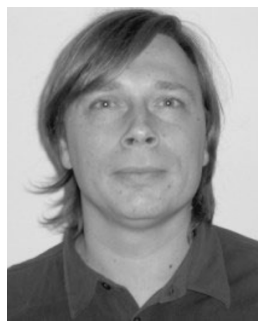

Guy Verschaffelt was born in Belgium in 1973. He received the M.E. degree in photonics and the Ph.D. degree from the Vrije Universiteit Brussels (VUB), Brussels, Belgium in 1996 and 2000, respectively.

Presently, he is a Postdoctoral Researcher with VUB. His research interests include polarization and noise properties of vertical-cavity surface-emitting lasers and microchip Nd:YAG lasers, emission properties of broad-area high-power semiconductor lasers, and the study of semiconductor ring lasers.

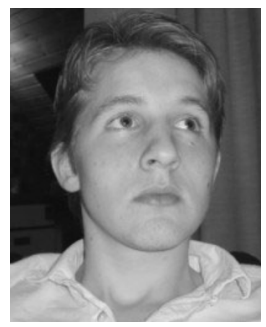

Gordon Craggs was born in Ukkel, Belgium, in 1985. He received the M.Sc. degree in physics from Vrije Universiteit Brussels (VUB), Brussels, Belgium, in 2007, where he is currently working toward the Ph.D. degree.

$\mathrm{He}$ is currently performing research on coherence properties of semiconductor lasers, presently more restricted to broad-area vertical-cavity surface-emitting lasers.

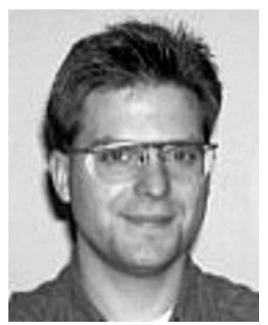

Michael L. F. Peeters was born in Antwerp, Belgium, in 1971. He received the M.E. degree in applied physics and photonics and the Ph.D. degree from the Vrije Universiteit Brussel (VUB), Brussels, Belgium, in 1995 and 2003, respectively. His doctoral work focused on vertical-cavity surface-emitting lasers.

His research at VUB focused on all aspects of the emission properties of these semiconductor devices. In addition to his research activities, he was active in the development of new teaching methods for courses in quantum physics, solid-state physics, and electromagnetism. Today, he leads the Alcatel-Lucent Bell Labs Access Node Technology and DSL team, Antwerp, looking into the future of broadband access over the existing copper infrastructure.

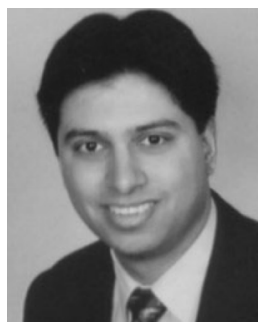

Shyam K. Mandre was born in Frankfurt, Germany, in 1977. He received the diploma degree in physics and the Ph.D. degree from Darmstadt University of Technology, Darmstadt, Germany, in 2002 and 2006, respectively.

His experimental research work focussed on the emission properties of high-power semiconductor lasers including multistripe lasers, broad-area lasers, and multimode VCSELs. He especially concentrated on the control of the spatiotemporal emission dynamics and spectral emission properties of both broad-area lasers and multimode VCSELs as well as on the coherence prop- erties of large-area VCSELs. Currently, he is with Procter and Gamble/Braun $\mathrm{GmbH}$, Kronberg, Germany, where he is with the R\&D Department.

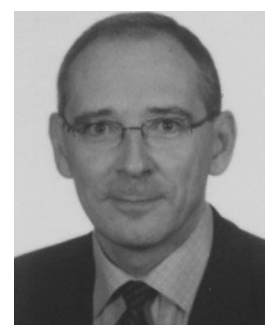

Hugo Thienpont (M'99) was born in Ninove, Belgium, in 1961. He graduated as an electrotechnical engineer and received the Ph.D. degree in applied sciences from the Vrije Universiteit Brussel (VUB), Brussels, Belgium, in 1984 and 1990, respectively.

In 1994, he became a Professor with the Faculty of Applied Sciences, VUB, with teaching responsibilities in photonics. In 2000, he became Research Director of the Department of Applied Physics and Photonics, VUB, and, in 2004, he was elected Chair of the department. Currently, he is the coordinator of several basic research and networking projects such as the European Network of Excellence on Micro-Optics (NEMO). In addition to academic-oriented research projects, he manages microphotonics-related industrial projects with companies like Barco, Agfa-Gevaert, Tyco, and Umicore. He authored more than 135 SCI-stated journal papers and more than 400 publications in international conference proceedings. He edited 15 conference proceedings and authored seven chapters in books. He was an invited speaker at 45 international conferences and is co-inventor of 13 patents.

Prof. Thienpont is a Fellow of SPIE and EOS and a member of the Optical Society of America and the IEEE Lasers and Electro-Optics Soceity (LEOS). He was Guest Editor of several special issues on Optical Interconnects for Applied Optics and the IEEE JouRnal OF SELECTED TOPICS ON QUANTUM ELECTRONICS and is General Chair of the SPIE Photonics Europe conferences in Strasbourg. In 1999, he was the recipient of the International Commission for Optics Prize ICO'99 and the Ernst Abbe Medal from Carl Zeiss. In 2003, he was awarded the title of "IEEE LEOS Distinguished Lecturer." He received the SPIE President's Award in 2005 for dedicated service to the European Community and the international MOC Award in 2007.

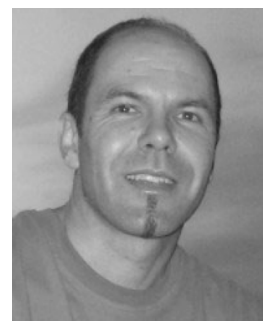

Ingo Fischer received the diploma and the Ph.D. degree in physics from Philipps University, Marburg, Germany, in 1992 and 1995, respectively.

After finishing his doctoral work, he was with Darmstadt University of Technology, Darmstadt, Germany, from 1995 to 2004 and with Vrije Universiteit Brussel, Brussels, Belgium, from 2005 to 2007. In addition, he has had research stays with Air Force Research Laboratories, Albuquerque, NM, in 1999, with ATR, Kyoto, Japan, in 1999 and 2000, and with UIB, n Palma de Mallorca, Spain in 2004 and 2007. Since 2007, he has been a full Professor of photonics with Heriot-Wat University, Edinburgh, U.K. His studies are focused on nonlinear photonics and brain dynamics. In particular, he is concentrating on emission properties and dynamics of modern photonic sources, coupled laser systems, synchronization of lasers and neurons, and utilization of chaos.

Dr. Fischer was the recipient of the Research Prize of the Adolf-Messer Foundation in 2000 and the first Hassian Cooperation Prize of the Technology Transfer Network in 2004. 